\title{
Immune checkpoints in aggressive breast cancer subtypes
}

\author{
I. ZAWLIK ${ }^{1,2, *, \neq}$, N. GABLO $^{1,2, \neq}$, B. SZYMANSKA ${ }^{3}$, Z. PAWLOWSKA ${ }^{3}$, C. CHUDOBINSKI ${ }^{4}$, J. CHALUBINSKA-FENDLER ${ }^{5}$, Z. MORAWIEC ${ }^{6}$, \\ H. ZIELINSKA-BLIZNIEWSKA ${ }^{7}$, A. MORAWIEC-SZTANDERA ${ }^{8}$, A. KOLACINSKA ${ }^{6,8}$
}

${ }^{1}$ Department of Genetics, Chair of Molecular Medicine, Faculty of Medicine, University of Rzeszow, Kopisto 2, 35-310 Rzeszow, Poland; ${ }^{2}$ Laboratory of Molecular Biology, Center for Innovative Research in Medical and Natural Sciences, University of Rzeszow, Warzywna 1A, 35-959 Rzeszow, Poland; ${ }^{3}$ Central Scientific Laboratory, Medical University of Lodz, Lodz, Poland; ${ }^{4}$ Department of Radiology, Cancer Center, Lodz, Poland; ${ }^{5}$ Department of Radiotherapy, Medical University of Lodz, Poland; ${ }^{6}$ Department of Surgical Oncology, Cancer Center, Lodz; Poland; ${ }^{7}$ Department of Allergology and Respiratory Rehabilitation, Medical University of Lodz, Poland; ${ }^{8}$ Department of Head and Neck Cancer Surgery, Medical University of Lodz; Poland

${ }^{*}$ Correspondence: izazawlik@yahoo.com

*Contributed equally to this work.

Received February 25, 2016 / Accepted May 4, 2016

\begin{abstract}
Immune checkpoints are molecules referred to inhibitory pathways in the immune system that play a pivotal role in prevention of autoimmunity and oncogenesis. The aim of the study was to evaluate expression levels of selected immune checkpoints- PD-1 (programmed cell death protein 1), and PD-L1 (programmed cell death 1 ligand 1) in breast cancer patients, suitable for breast conservation and sentinel node biopsy and determine their associations with clinicopathological factors.

Expression of the genes coding for PD-1 and PD-L1 was analyzed in formalin-fixed paraffin-embedded specimens using realtime PCR. mRNA expression levels were determined using beta actin (ACTB) as an endogenous control. There was a trend towards significance between higher PD-1 and PD-L1 levels in triple negative breast cancers $(\mathrm{p}=0.1)$. Higher PD-L1 expression was also found in aggressive breast cancer subtypes e.g. triple negative and HER2 (human epidermal growth factor receptor 2) -positive as compared with subtypes with better prognosis such as luminal A and luminal BHER2-negative ( $\mathrm{p}=0.05)$. There was a trend towards significance in higher PD-1 levels in triple negative and HER-2 positive breast cancers $(\mathrm{p}=0.1)$. A statistically significant difference was found between PD-L1 expression and tumor grade ( $\mathrm{p}=0.01)$. Elevated PD-L1 levels were noted in G3 tumors.

Immunogenicity appears to be gaining importance in triple negative and HER2-positive molecular subtypes of breast cancer, and the results in this study provide a basis for further investigation into the role of immune checkpoints in breast cancer.
\end{abstract}

Key words: immune checkpoints, breast cancer, PD-1, PD-L1

Breast cancer is a major cause of cancer-related death among women worldwide [1]. Currently breast cancer is one of the major cancer types for which new immune-based cancer treatments are in development [2]. Triple-negative breast cancer (TNBC), is characterized by a lack of expression of estrogen receptor (ER), progesterone receptor (PR), and HER2/ neu. TNBCs are generally high-grade, aggressive tumors with

\footnotetext{
Abbreviations: ACTB - beta actin; CTLA-4 - cytotoxic T-lymphocyte antigen 4; ER - estrogen receptor; FFPE formalin-fixed paraffin-embedded; HER2 - human epidermal growth factor receptor 2; IBC - inflammatory breast cancer; PD-1 - programmed cell death protein 1; PD-L1 - programmed cell death 1 ligand; $\mathrm{PR}$ - progesteron receptor
}

a high rate of distant metastasis and limited treatment options [3]. Novel therapeutic strategies are needed to improve the management of patients with TNBC. A promising avenue of clinical research in breast cancer is the use of immune checkpoints. Immune checkpoints are molecules referred to inhibitory pathways in the immune system that a pivotal role in prevention of autoimmunity, modulation of the duration and amplitude of physiological immune responses to ensure that is not excessive [4]. Recent research has underlined their role in oncogenesis [5]. CTLA-4 (cytotoxic T-lymphocyte antigen 4, also known as cluster of differentiation CD152)the godfather of immune checkpoints, expressed on a surface of $\mathrm{T}$ cells negatively regulating the immune system. CTLA- 4 display termination activity for T cells attack on the antigen 
in early stages of immune response phase, mainly by naïve cells. Significant overexpression of CTLA- 4 is associated with immune tolerance against tumor cells. CTLA-4 is operational during early activation of $\mathrm{T}$ naïve cells in lymphatic tissues. One of the typical features of CTLA- 4 blockade is the durability of objective tumor response $[4,6,7]$. PD-1 (programmed cell death protein 1 , also known as cluster of differentiation CD279)- is an immune inhibitory receptor expressed on the surface of activated T cells, B cells, natural killers and macrophages and it has two ligands PD-L1 and L2. The interaction of PD-1 with its two ligands occurs in limited effector T-cell activity in peripheral tissue, including the tumor itself and its microenvironment and leads to apoptosis [8].

PD-L1 (programmed cell death 1 ligand 1, also known as cluster of differentiation CD274) is expressed on a wide variety of normal tissues including natural killer cells, macrophages, B cells, epithelial cells. Its normal physiologic role is to bind PD-1 receptors expressed on the surface of activated cytotoxic $\mathrm{T}$ cells. This binding causes inhibition of $\mathrm{T}$ cell activation. The PD-1/PD-L1 interaction serves as an important regulatory check against an excessive adoptive immune response to antigens and autoimmunity. The expression of PD-L1 has been evaluated in numerous tumors including breast cancer $[5,6$, 9]. It has been observed that there was a positive correlation between Real-Time PCR and immunohistochemistry staining method for PD-L1 expression indicates that these two methods can be used independently [10].

Inhibitors application for immune checkpoints receptors such as CTLA-4 and PD-1, has emerged as successful immunotherapeutic drugs for patients with advanced melanoma, non-small cell lung cancer and renal cell carcinoma (e.g. anti-CTLA-4 - ipilimumab, anti-PD1 - nivolumab, pembrolizumab) $[11,12]$.

Unlike other solid tumor types, such as melanoma with elevated mutational load, thus activating an antitumor immune response, breast cancer has not traditionally been thought to be immunogenic [13]. But recent studies on tumor-infiltrating lymphocytes, immune milieu, checkpoints and significant heterogeneity within breast cancer subtypes, have cast new light on the role of immune system in breast cancer [14].

The aim of the study was to evaluate expression levels of PD-1 and PD-L1 immune checkpoints in formalin- fixed, paraffinembedded breast cancer samples and determine their associations with clinicopathological features e.g. age, tumor size, grade, surrogates of molecular subtypes and sentinel node status.

\section{Patients and methods}

Inclusion criteria. We enrolled 50 patients with operable breast cancer, stages I-II, suitable for breast conservative and negative surgery sentinel node biopsy, and operated on in the Department of Surgical Oncology. Archival formalin- fixed, paraffin-embedded tumor samples were used for PD-1 and PD-L1 assessment. All samples were taken with written informed consent. Ethics committee approval was obtained from the Institutional Review Board of the Medical University of Lodz (Number RNN/239/13/KE).

Exclusion criteria. We ruled out breast cancer patients with concomitant or previous autoimmune diseases, other immune aberrations or a medical history of any malignancy. Pregnant or lactating women were also excluded from the studies.

\section{PD-1 and PD-L1 assessment}

Total RNA isolation. Total RNA was extracted from formalin-fixed paraffin-embedded (FFPE) tissue using the Roche High Pure miRNA Isolation Kit according to the manufacturer's instruction. In brief, FFPE slices were processed in a $2 \mathrm{ml}$ Eppendorf tubes, deparaffinized with $100 \%$ xylene, followed by wash with $100 \%$ ethanol and dried at $55^{\circ} \mathrm{C}$ for 10 min. The dry tissue was resuspended in Paraffin Tissue Lysis Buffer (included in the kit) and digested with proteinase $\mathrm{K}$ at $55^{\circ} \mathrm{C}$ overnight. Subsequent steps of RNA purification on column were performed following the manufacturer's recommendations. The yield and quality (the ratio of absorptions at 260/280 nm) of RNA product were measured using PicoDrop spectrophotometer (Picodrop Limited, UK). The purified total RNA was immediately used for cDNA synthesis or stored at $-80^{\circ} \mathrm{C}$ until use.

cDNA generation. Generation of cDNA was performed with High Capacity cDNA Reverse Transcription Kits (Applied Biosystems) according to protocol of the manufacturer. $1 \mu \mathrm{g}$ of total RNA was used as starting material, to which was added $2 \mathrm{x}$ RT master mix containing $2 \mu \mathrm{l}$ of $10 \mathrm{x}$ RT Buffer, $0.8 \mu \mathrm{l}$ of 25x dNTP Mix (100 mM), $2 \mu \mathrm{l}$ of 10x RT Random Primers, 1 $\mu \mathrm{l}$ MultiScribe ${ }^{\mathrm{m}}$ Reverse Transcriptase and $1 \mu \mathrm{l}$ RNase Inhibitor per each $20 \mu \mathrm{L}$ reaction. Reverse transcription was performed under conditions optimized for use this kit $\left(25^{\circ} \mathrm{C}\right.$ for $10 \mathrm{~min}$, $37^{\circ} \mathrm{C}$ for $120 \mathrm{~min}, 85^{\circ} \mathrm{C}$ for $\left.5 \mathrm{~min}\right)$. The samples were kept frozen at $-20 \mathrm{C}$.

Real Time PCR analysis. Measurement of mRNA expression was done using standard TaqMan ${ }^{\odot}$ Gene Expression Assays (Applied Biosystems): programmed cell death 1 (PDCD1, Hs01550088_m1), and its ligand PD-L1, CD274 molecule (CD274, Hs01125301_m1) and beta actin (ACTB, Hs01060665_g1) as the endogenous control. TaqMan PCR assays were performed in $10 \mu \mathrm{l}$ reactions included $50 \mathrm{ng}$ cDNA, $5 \mu$ KAPA PROBE FAST qPCR Kit Master Mix ABI Prism (Kapa Biosystems) and 0,5 $\mu$ l appropriate TaqMan Gene Expression Assay. All reactions were run in duplicate on a 7900HT Fast Real-Time PCR System (Applied Biosystems) in 96 well PCR plates. The following thermal cycling specifications were performed: $20 \mathrm{~s}$ at $95^{\circ} \mathrm{C}$ and 40 cycles each for $30 \mathrm{~s}$ at $95^{\circ} \mathrm{C}$ and $30 \mathrm{~s}$ at $60^{\circ} \mathrm{C}$. Data was analyzed using SDS 2.4 software (Applied Biosystems).

Statistical analysis. Continuous variables were presented as medians and interquartile ranges. Expression levels were shown as dCt values - calculated using the standard formula, where higher $\mathrm{dCt}$ represents lower expression score [15]. Due to significant deviation from normal distribution (evaluated with the Shapiro-Wilk's test), non-parametric tests were used to confirm statistical significance. Mann-Whitney's U test 

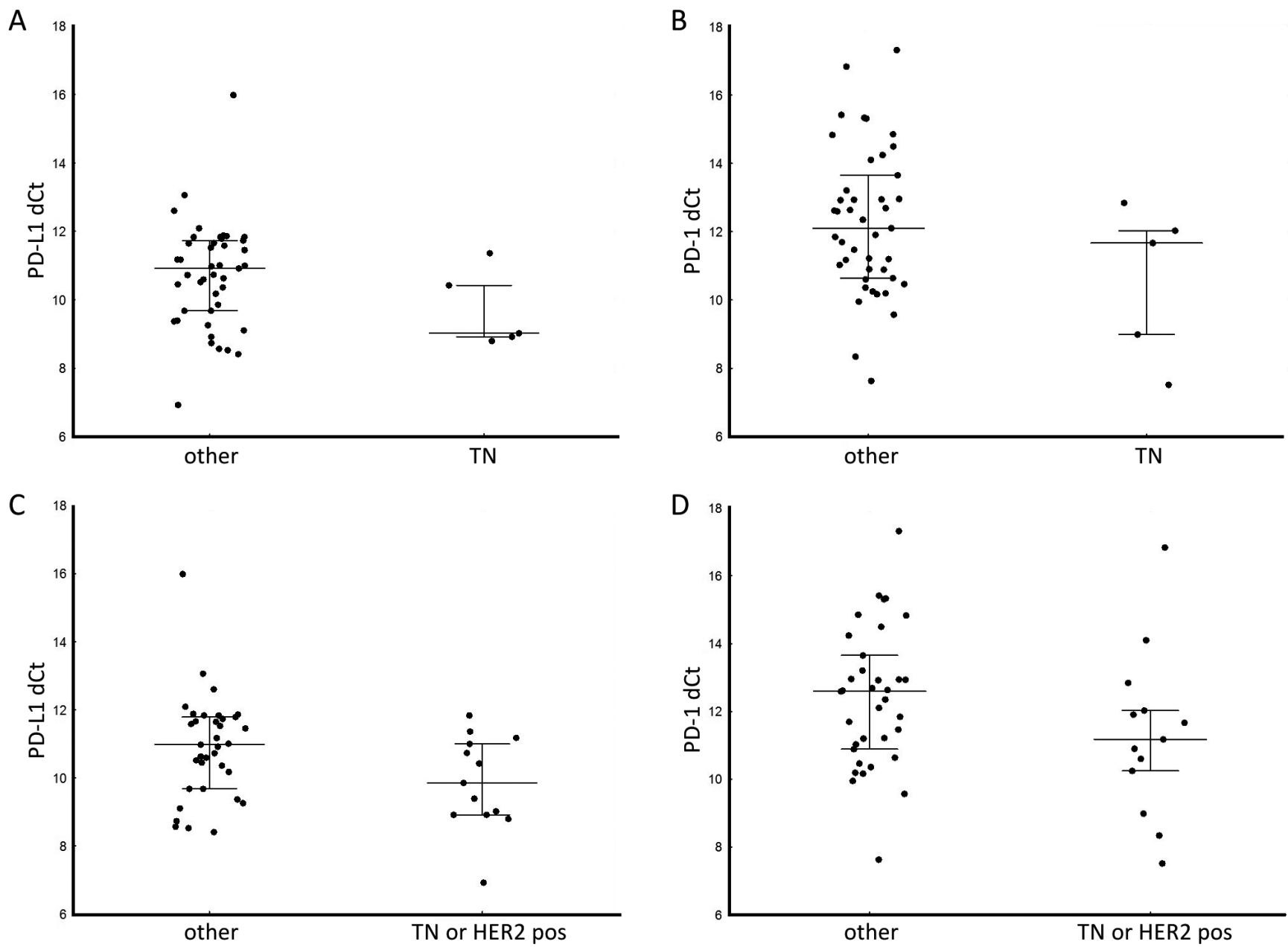

Figure 1. (A) Association between PD-L1 expression level and breast cancer subtype (triple negative versus luminal A and luminal B HER2 negative subtypes $\mathrm{p}=\mathbf{0 . 1}$ ). Higher PD-L1 expression was found in triple negative breast cancer subtype. Expression levels were shown as dCt values - calculated using the standard formula, where higher dCt represents lower expression score [20]. PD-L1-programmed cell death 1 ligand 1. (B) Association between PD-1 expression level and breast cancer subtype ((triple negative versus luminal A and luminal B HER2 negative subtypes p=0.1). Higher PD-1 expression was found in triple negative breast cancer subtype. Expression levels were shown as dCt values - calculated using the standard formula, where higher dCt represents lower expression score [20]. PD-1- programmed cell death protein 1. (C) Association between PD-L1 expression level and breast cancer subtype (triple negative and HER2-positive versus luminal A and luminal BHER2 negative subtypes) $\mathrm{p}=0.05$. Higher PD-L1 expression was found in triple negative and HER2-positive breast cancer subtypes. Expression levels were shown as dCt values - calculated using the standard formula, where higher dCt represents lower expression score [20]. PD-L1-programmed cell death 1 ligand 1. (D) Association between PD-1 expression level and breast cancer subtype (triple negative and HER2-positive versus luminal A and luminal BHER2 negative subtypes) $p=0.1$. Higher PD-1 expression was found in triple negative and HER2-positive breast cancer subtypes. Expression levels were shown as dCt values - calculated using the standard formula, where higher dCt represents lower expression score [20]. PD-1- programmed cell death protein 1

was used for two group comparisons, Kruskal-Wallis test for multiple group comparisons and Spearman's rank correlation test for correlation assessment. Values of p lower than 0.05 were considered as statistically significant.

\section{Results}

Detailed patient characteristics are shown in Table 1.

We did not find a correlation between immune checkpoint expression in paraffin-embedded tumor samples and patients' age, tumor size and nodal status.
Higher PD-1 and PD-L1 levels were noted in triple negative breast cancers than in other groups, but the difference failed to reach statistical significance $(\mathrm{p}=0.1)$ (Figure 1A and 1B). Higher PD-L1 expression was also found in aggressive breast cancer subtypes e.g. triple negative and HER2-positive as compared with subtypes with better prognosis such as luminal A and luminal BHER2-negative $(\mathrm{p}=0.05)$ (Figure 1C). Similarly, PD-1 levels were higher in triple negative and HER-2 positive breast than luminal A and luminal B HER2-negative cancers, although the difference did not reach statistical significance $(\mathrm{p}=0.1)$ (Figure 1D). A statistically significant 
difference was found between PD-L1 expression and tumor grade with grade 3 showing significantly higher expression levels than grade 1 and 2 ( $p=0.01$ in ANOVA and $p<0.05$ in pairwise post-hoc comparisons) (Figure 2).

\section{Discussion and conclusions}

The immunogenicity of breast cancer is an emerging area of research, and highlighted at the ASCO meeting (American Society of Clinical Oncology) in May/ June 2015, St. Gallen Early Breast Cancer Conference in March 2015 and San Antonio Breast Cancer Symposium in December 2014 [13]. Loi et al. indicated that breast cancer itself does not have mutational capacity leading to immunogenic responses seen in other tumor types, although it is generally higher in the triple-negative and HER2-positive groups, as compared with the luminal breast cancers [9]. This is in concordance with our research demonstrating higher PD-1 and PD-L1 expression in aggressive phenotypes such as triple negative and HER2-positive as compared with luminal A and luminal B HER2-negative breast cancers. We also showed that elevated PD-L1 expression was more noticeable in high grade tumors. Bertucci et al. demonstrated that PD-L1 expression was higher in aggressive inflammatory breast cancer (IBC) than in non-IBC. In IBC, PD-L1 overexpression was associated with estrogen receptor-negative status, basal and ERBB2-enriched aggressive subtypes. Moreover, these authors indicated that PD-L1 overexpression was associated with better pathological response to chemotherapy and may be a biomarker for patient selection for immunogenic chemotherapy [16]. In our research we only enrolled patients with early stage breast cancer, so we were unable to assess the pathologic response to chemotherapy in the neoadjuvant setting. Sabatier et al. revealed that high PD-L1 expression was associated with poor-prognosis features (high grade, ER-negative, PRnegative, ERBB2-positive status, high proliferation, basal and ERBB2-enriched subtypes). These authors concluded that reactivation of dormant tumor-infiltrating lymphocytes by PDL1-inhibitors could represent promising strategy in PDL1-upregulated basal breast cancer [17]. These findings were also confirmed by Soliman et al. who showed that basal/ triple negative breast cancer cell lines expressed the highest levels of PD-L1 [18].

Disis et al. further substantiated the immunogenicity of triple negative breast cancer, demonstrating an amplification of the adaptive immune response through B-cell pathways with antibody secretion binding to tumor antigens [19].

In the current study we have examined immune biomarker expression in archival paraffin-embedded tumor samples, but in our previous research described elsewhere we found a striking difference $(\mathrm{p}<0.0001)$ between immune checkpoint $\mathrm{PD}-1$ expression in CD8+ T cells in blood samples of breast cancer patients and healthy controls, with significantly lower levels in the latter group [20]. Moreover, in our previous study, there was a negative correlation between $\mathrm{PD}-1$ expression and

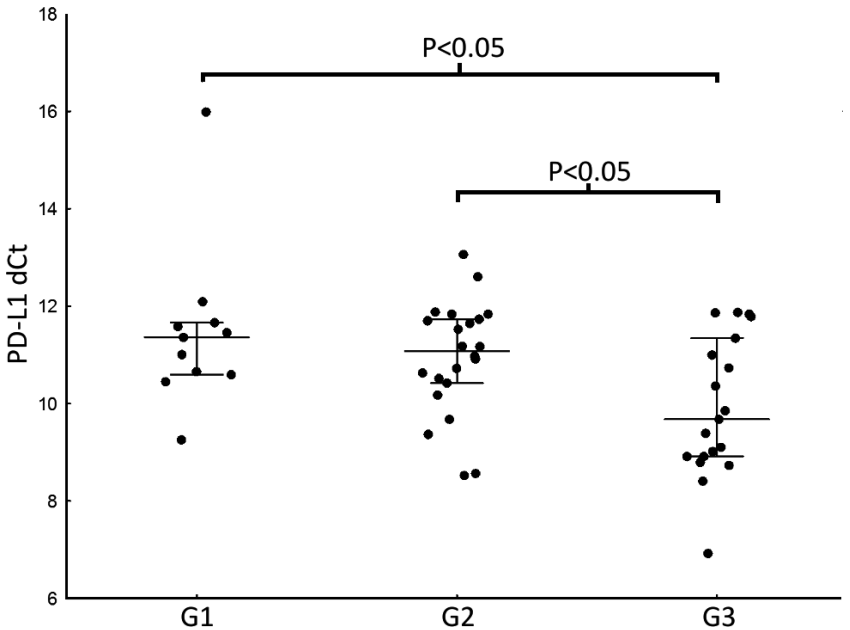

Figure 2. Association between PD-L1 expression level and tumor grade $(\mathbf{p}=0.01)$. Higher PD-L1 expression was found in G3 tumors. Expression levels were shown as $\mathrm{dCt}$ values - calculated using the standard formula, where higher $\mathrm{dCt}$ represents lower expression score [20]. PD-L1-programmed cell death 1 ligand 1

Table 1. Clinical characteristics of the study group $(\mathrm{N}=52)$

\begin{tabular}{|c|c|}
\hline $\begin{array}{l}\text { Age, years } \\
\text { median (range) }\end{array}$ & $\begin{array}{l}59.7 \\
(31-82)\end{array}$ \\
\hline Tumor type & $\begin{array}{l}\text { NST- } 45 \\
\text { Lobular- } 5 \\
\text { Mucinous- } 1 \\
\text { Papillary- } 1\end{array}$ \\
\hline Tumor grade & $\begin{array}{l}\text { G1-11 } \\
\text { G2- } 22 \\
\text { G3- } 19\end{array}$ \\
\hline Pathologic tumor size & $\begin{array}{l}\text { pT1a- } 2 \\
\text { pT1b- } 3 \\
\text { pT1c- } 25 \\
\text { pT2- } 22\end{array}$ \\
\hline Sentinel node status & $\begin{array}{l}\text { pN0- } 36 \\
\text { pN1mi- } 5 \\
\text { pN1- } 11\end{array}$ \\
\hline Receptor status & $\begin{array}{l}\text { ER positive- } 40 \\
\text { ER- negative- } 10 \\
\text { ER-unknown-2 } \\
\text { PR-positive- } 35 \\
\text { PR- negative- } 15 \\
\text { PR-unknown-2 } \\
\text { HER2-positive- } 8 \\
\text { HER2-negative- } 40 \\
\text { HER2-unknown- } 4\end{array}$ \\
\hline Surrogates of molecular subtypes & $\begin{array}{l}\text { Luminal A- } 16 \\
\text { Luminal BHER2 negative- } 19 \\
\text { Luminal BHER2 positive- } 5 \\
\text { Non-luminal HER2-positive- } 3 \\
\text { Triple Negative- } 5 \\
\text { unknown- } 4\end{array}$ \\
\hline
\end{tabular}


progesterone receptor (PR) status in blood of breast cancer patients $(\mathrm{p}=0.024)$. The lack of $\mathrm{PR}$, associated with the poor prognosis triple negative subtype, correlated with higher PD-1 expression, and thus augmented inhibition of the immune system. This is consistent with our current study in which we found higher PD-1 and PD-L1 expression in aggressive breast cancer subtypes e.g. triple negative and HER2-positive in contrast to subtypes with better prognosis such as luminal A and luminal BHER2-negative. In view of our small sample size these results should be considered preliminary and require further elucidation. However, they provide a basis for further investigation into the role of immune checkpoints in breast cancer, especially in aggressive phenotypes, and potentially also justification for more personalized therapies in breast cancer patients on immunological grounds, to enhance the effects of the other already well-established multimodality treatment- chemotherapy, and anti-HER2 therapy [13]. Nanda et al. presented the first report of clinical activity of an immune checkpoint inhibitor-pembrolizumab, in triple negative breast cancer in a group of pre-treated patients with recurrent/ metastatic disease in a phase Ib study [21]. Pembrolizumab is a highly selective, humanized IgG4/kappa isotype $\mathrm{mAb}$ created to stop PD-1 interaction with its ligands PD-L1 and PD-L2, thereby stimulating the immune system to eliminate cancer [21].

Emens et al. demonstrated a 19\% objective response rate to the PD-L1 inhibitor MPDL3280A (9.5\% of complete and $9.5 \%$ of partial response) with ongoing responses in $75 \%$ of pretreated patients with metastatic triple negative breast cancer [22]. Loi et al. added that agents targeting the PD-1/PD-L1 pathways look promising in the more aggressive subtypes of breast cancer [13]. Even if the response is limited to a small proportion of patients, it seems to be durable $[13,22]$.

Therefore, performing "immune profiling" of the tumor, including immune gene signatures, evaluation of TILs and immune checkpoints assessment in tumor, its microenvironment, as well as immune profiling of the peripheral blood, will help us understand the differences between responders and non-responders $[13,23]$. The role of immunotherapy in breast cancer patients should become clearer with time [13, 24]. Hence, further investigation in larger studies is urgently required. The International Breast Cancer Study Group IBCSG $45-13$ is currently conducting a phase Ib/II trial of anti-PD-1 monoclonal ANtibody in AdvanCed. Trastuzumab-resistant, HER2-positive breast cAncer, PD-L1-positive (PANACEA), to evaluate the efficacy of MK-3475 and trastuzumab in patients with HER2-positive metastatic breast cancer [25]. During the $14^{\text {th }}$ St. Gallen International Breast Cancer Conference Primary Therapy of Early Breast Cancer, March, 2015, Curigliano suggested including TILs and immune checkpoints in pathology reports, especially in the more immunogenic breast cancer phenotypes such as triple negative and HER2-positive [26].

In conclusion, breast cancer has not been considered to be immunogenic. However, immunogenicity appears to be gaining importance in triple negative and HER2-positive molecular subtypes of breast cancer, and the results in this study provide a basis for further investigation into the role of immune checkpoints in breast cancer.

Acknowledgements: This study was supported by the grant of the National Science Center, Poland, no. 2011/01/B/NZ4/03345. The authors would like to thank Professor Andrew Shorthouse, the Emeritus Professor of Surgery, University of Sheffield, for proofreading the manuscript. The study was partly performed within the project 'Centre for Innovative Research in Medical and Natural Sciences' realized by University of Rzeszow, co-financed within Regional Operational Programme for the Podkarpackie Province for the years 2007-2013, contract number UDA-RPPK.01.03.00-18-004/12-00.

\section{References}

[1] SIEGEL R, MA J, ZOU Z, JEMAL A. Cancer statistics, 2014. CA Cancer J Clin 2014; 64: 9-29. http://dx.doi.org/10.3322/ caac. 21208

[2] PUSZTAI L, KARN T, SAFONOV A, ABU-KHALAF MM, BIANCHINI G. New Strategies in Breast Cancer: Immunotherapy. Clin Cancer Res 2016; 22: 2105-2110. http://dx.doi. org/10.1158/1078-0432.CCR-15-1315

[3] HUDIS CA, GIANNI L. Triple-negative breast cancer: an unmet medical need. Oncologist 2011; 16 Suppl 1: 1-11. http:// dx.doi.org/10.1634/theoncologist.2011-S1-01

[4] PARDOLL DM. The blockade of immune checkpoints in cancer immunotherapy. Nat Rev Cancer 2012; 12: 252-264. http://dx.doi.org/10.1038/nrc3239

[5] TOPALIAN SL, WOLCHOK JD, CHAN TA, MELLMAN I, PALUCKA $\mathrm{K}$ et al. Immunotherapy: The path to win the war on cancer? Cell 2015; 161: 185-186. http://dx.doi. org/10.1016/j.cell.2015.03.045

[6] MAHONEY KM, RENNERT PD, FREEMAN GJ. Combination cancer immunotherapy and new immunomodulatory targets. Nat Rev Drug Discov 2015; 14: 561-584. http://dx.doi. org $/ 10.1038 / \mathrm{nrd} 4591$

[7] LI F, GUO Z, YU H, ZHANG X, SI T et al. Anti-tumor immunological response induced by cryoablation and antiCTLA-4 antibody in an in vivo RM-1 cell prostate cancer murine model. Neoplasma 2014; 61: 659-671. http://dx.doi. org/10.4149/neo $2014 \quad 081$

[8] TOPALIAN SL, HODI FS, BRAHMER JR, GETTINGER SN, SMITH DC et al. Safety, activity, and immune correlates of anti-PD-1 antibody in cancer. N Engl J Med 2012; 366: 2443-2454. http://dx.doi.org/10.1056/NEJMoa1200690

[9] BRAHMER JR, TYKODI SS, CHOW LQ, HWU WJ, TOPALIAN SL et al. Safety and activity of anti-PD-L1 antibody in patients with advanced cancer. N Engl J Med 2012; 366: 2455-2465. http://dx.doi.org/10.1056/NEJMoa1200694

[10] OHIGASHI Y, SHO M, YAMADA Y, TSURUI Y, HAMADA $\mathrm{K}$ et al. Clinical significance of programmed death-1 ligand-1 and programmed death-1 ligand-2 expression in human esophageal cancer. Clin Cancer Res 2005; 11: 2947-2953. http:// dx.doi.org/10.1158/1078-0432.CCR-04-1469

[11] MCDERMOTT DF, DRAKE CG, SZNOL M, CHOUEIRI TK, POWDERLY JD et al. Survival, Durable Response, 
and Long-Term Safety in Patients With Previously Treated Advanced Renal Cell Carcinoma Receiving Nivolumab. J Clin Oncol 2015; 33: 2013-2020. http://dx.doi.org/10.1200/ LCO.2014.58.1041

[12] ROBERT C, LONG GV, BRADY B, DUTRIAUX C, MAIO M et al. Nivolumab in previously untreated melanoma without BRAF mutation. N Engl J Med 2015; 372: 320-330. http:// dx.doi.org/10.1056/NEJMoa1412082

[13] Loi S. Immune targeting in breast cancer: in whom and with what? Oncology (Williston Park) 2015; 29: 386-387.

[14] HUA Z, LI D, XIANG G, XU F, JIE G et al. PD-1 polymorphisms are associated with sporadic breast cancer in Chinese Han population of Northeast China. Breast Cancer Res Treat 2011; 129: 195-201. http://dx.doi.org/10.1007/s10549-011-1440-3

[15] LIVAK KJ, SCHMITTGEN TD. Analysis of relative gene expression data using real-time quantitative PCR and the 2(-Delta Delta C(T)) Method. Methods 2001; 25: 402-408. http://dx.doi.org/10.1006/meth.2001.1262

[16] BERTUCCI F, FINETTI P, COLPAERT C, MAMESSIER E, PARIZEL $M$ et al. PDL1 expression in inflammatory breast cancer is frequent and predicts for the pathological response to chemotherapy. Oncotarget 2015; 6: 13506-13519. http:// dx.doi.org/10.18632/oncotarget.3642

[17] SABATIER R, FINETTI P, MAMESSIER E, ADELAIDE J, CHAFFANET $M$ et al. Prognostic and predictive value of PDL1 expression in breast cancer. Oncotarget 2015; 6: 5449-5464. http://dx.doi.org/10.18632/oncotarget.3216

[18] SOLIMAN H, KHALIL F, ANTONIA S. PD-L1 expression is increased in a subset of basal type breast cancer cells. PLoS One 2014; 9: e88557. http://dx.doi.org/10.1371/journal. pone. 0088557

[19] DISIS ML, STANTON SE. Triple-negative breast cancer: immune modulation as the new treatment paradigm. Am Soc Clin Oncol Educ Book 2015; e25-30.

[20] KOLACINSKA A, CEBULA-OBRZUT B, PAKULA L, CHALUBINSKA-FENDLER J, MORAWIEC-SZTANDERA A et al. Immune checkpoints: Cytotoxic T-lymphocyte antigen 4 and programmed cell death protein 1 in breast cancer surgery. Oncol Lett 2015; 10: 1079-1086. http://dx.doi.org/10.3892/ ol.2015.3321

[21] NANDA R, CHOW LQ, DEES EC, BERGER R, GUPTA S ET AL. Pembrolizumab in Patients With Advanced TripleNegative Breast Cancer: Phase Ib KEYNOTE-012 Study. J Clin Oncol 2016; pii: JCO648931, ahead of print http://dx.doi. org/10.1200/JCO.2015.64.8931

[22] EMENS LA, BRAITEH FS, CASSIER P, DELORD JP, EDER JP et al. Inhibition of PD-L1 by MPDL3280A leads to clinical activity in patients with metastatic triple-negative breast cancer. Cancer Res 2015; 75: Abstract PD1-6

[23] SALGADO R, DENKERT C, CAMPBELL C, SAVAS P, NUCIFORO $P$ et al. Tumor-Infiltrating Lymphocytes and Associations With Pathological Complete Response and Event-Free Survival in HER2-Positive Early-Stage Breast Cancer Treated With Lapatinib and Trastuzumab: A Secondary Analysis of the NeoALTTO Trial. JAMA Oncol 2015; 1: 448-454. http://dx.doi.org/10.1001/ jamaoncol.2015.0830

[24] DENKERT C, VON MINCKWITZ G, BRASE JC, SINN BV, GADE $S$ et al. Tumor-infiltrating lymphocytes and response to neoadjuvant chemotherapy with or without carboplatin in human epidermal growth factor receptor 2-positive and triple-negative primary breast cancers. J Clin Oncol 2015; 33: 983-991. http://dx.doi.org/10.1200/JCO.2014.58.1967

[25] HTTPS://CLINICALTRIALS.GOV/CT2/SHOW/NCT021 29556? TERM=PANACEA\&RANK=2.

[26] ESPOSITO A, CRISCITIELLO C, CURIGLIANO G. Highlights from the 14(th) St Gallen International Breast Cancer Conference 2015 in Vienna: Dealing with classification, prognostication, and prediction refinement to personalize the treatment of patients with early breast cancer. Ecancermedicalscience 2015; 9: 518. http://dx.doi.org/10.3332/ ecancer.2015.518 